Ethics Approval The study protocols for clinical trials represented in this publication were reviewed by the respective IRB/IEC at each study site and approved before trial participants were screened and enrolled.

Consent Not applicable.

http://dx.doi.org/10.1136/jitc-2020-SITC2020.0323

\section{BDB001, A TOLL-LIKE RECEPTOR 7 AND 8 (TLR7/8) AGONIST, CAN BE SAFELY ADMINISTERED INTRAVENOUSLY AND SHOWS CLINICAL RESPONSES IN ADVANCED SOLID TUMORS}

${ }^{1}$ Manish Patel ${ }^{*},{ }^{2}$ Drew Rasco, ${ }^{3}$ Melissa Johnson, ${ }^{4}$ Anthony Tolcher, ${ }^{5}$ Lixin Li, ${ }^{5}$ Adam Zong, ${ }^{5}$ Alexander Chung, ${ }^{5}$ Robert Andtbacka. ${ }^{1}$ Florida Cancer Specialists and Research, Sarasota, FL, USA; ${ }^{2}$ START Center for Cancer Care, San Antonio, TX, USA; ${ }^{3}$ Sarah Cannon Cancer Center, Nashville, TN, USA; ${ }^{4}$ NEXT Oncology, San Antonio, TX, USA; ${ }^{5}$ Seven and Eight Biopharmaceuticals inc., Edison, NJ, USA

Background TLR agonists mediate antitumor activity through dendritic cell (DC) activation. Most TLR agonists in development are administered intratumorally allowing for less than $30 \%$ of advanced solid tumor to be treated. BDB001 is an intravenously administered novel TLR7/8 agonist that activates plasmacytoid and myeloid DCs and has shown to have activity in preclinical studies. Here we report on BDB001 administration in patients with advanced solid tumors.

Methods BDB001-101 is a Phase 1, open label, dose escalation/expansion trial of BDB001 administered intravenously weekly in patients with advanced solid tumors. The primary endpoint was safety and tolerability. Secondary endpoints included efficacy, pharmacokinetics and pharmacodynamic profiling of immune activation.

Results Thirty-six subjects with 16 different tumor types were enrolled across 5 dose levels. Sixty seven percent were female, median age was 66 years (range, 38-88), median number of prior therapies was 4 (range, $0-12$ ), and $61 \%$ of tumors had progressed on prior anti-PD-(L)1 therapy. BDB001 was well tolerated and a maximum tolerated dose was not reached. Eleven (30.5\%) subjects had no treatment related adverse events (AEs) and the majority of AEs were Grade 1 or 2. Three (8.3\%) subjects had Grade 3 AEs, including 2 with a cytokine release syndrome, both of whom were clinically stable and had symptoms fully resolved within 2 to 5 days. There were no Grade 4 or 5 AEs. The most common AEs included chills/rigor (19.4\%), fever (19.4\%), fatigue (11.1\%), nausea (11.1\%) and pruritus (11.1\%). Of 32 subjects evaluable for efficacy, best overall response rate was: $6 \%$ durable partial response, $56 \%$ stable disease, $38 \%$ progressive disease, for a disease control rate of $62 \%$. Durable responses were seen in renal cell carcinoma and non-small cell lung cancer. Interestingly, clinical activity favored subjects with tumors that had progressed on prior anti-PD-(L)1 therapy, compared to prior DNA-damaging chemotherapy, within 6 months of BDB001 initiation. Median time on treatment was 12.1 weeks (range, 3.1 - 68.0). Transcriptional profiling showed up-regulation of interferon inducible genes, activation of dendritic cells and macrophages. BDB001 also significantly increased serum levels of interferon gamma and interferon inducible protein-10 (IP10).

Conclusions Intravenously administered BDB001 monotherapy was well tolerated. Clinical responses were achieved, supported by BDB001-induced immune activation. Preliminary findings suggest that BDB001 is a promising therapeutic option for patients with tumors that progress on anti-PD-(L)1 therapy. BDB001 is also being evaluated in combination with pembrolizumab (anti-PD-1, NCT03486301) and with atezolizumab (anti-PD-L1, NCT04196530).

Trial Registration NCT03486301

Ethics Approval This study was approved by the institutional review boards at the four participating institutions. All subjects signed informed consent before enrolling in the clinical trial.

http://dx.doi.org/10.1136/jitc-2020-SITC2020.0324

\section{IMMUNOTHERAPY WITH B CELL ACTIVATING ANTIBODY CPI-006 IN PATIENTS (PTS) WITH MILD TO MODERATE COVID-19 STIMULATES ANTI-SARS-COV-2 ANTIBODY RESPONSE, MEMORY B CELLS AND MEMORY T EFFECTOR CELLS}

${ }^{1}$ Gerard Criner* ${ }^{2}$ Mehrdad Mobasher, ${ }^{2}$ Craig Hill, ${ }^{2}$ Shenshen Hu, ${ }^{2}$ Suresh Mahabhashyam, ${ }^{3}$ Joshua Brody, ${ }^{3}$ Thomas Marron, ${ }^{2}$ Stephen Willingham, ${ }^{2}$ Richard Miller. ${ }^{1}$ Temple University Hospital, Philadelphia, USA; ${ }^{2}$ Corvus Pharmaceuticals Inc, Burlingame, CA, USA; ${ }^{3}$ /cahn School of Medicine at Mount Sinai, New York, NY, USA

Background CD73 is present on subsets of B and T cells and is involved in lymphocyte activation. CPI-006 is a humanized IgG1, Fcy receptor deficient anti-CD73 that has agonistic properties. In vitro studies and ongoing cancer clinical trials show that CPI-006 binds to B cells leading to expression of CD69, trafficking to lymph nodes, immunoglobulin class switching, transformation to plasmablasts and generation of memory B cells. ${ }^{1}$ Recently, a patient in the cancer trial with asymptomatic COVID-19 developed high titers of neutralizing anti-SARS-CoV-2 antibodies following administration of CPI006. A phase 1 trial in COVID-19 was initiated to evaluate the use of CPI-006 to enhance anti-viral immune response (NCT04464395).

Methods Single intravenous dose escalation with $\mathrm{N}=5$ per cohort of $0.3,1.0,3.0$ and $5.0 \mathrm{mg} / \mathrm{kg}$. Pt eligibility included PCR positive nasal swab for COVID-19; hospitalized with $\mathrm{O}_{2}$ saturation of $\geq 92 \%$ on $<5 \mathrm{l} / \mathrm{min}$ of $\mathrm{O}_{2}$. Pts received standard care for COVID-19. Pts were monitored for safety, COVID19 symptoms, inflammatory markers and anti-SARS-CoV-2 antibodies by ELISA. Immunophenotyping of blood by flow cytometry was performed.

Results 10 pts have been treated in the first 2 cohorts; median age 64 (range 28-76) and all had comorbidities: diabetes (4), hypertension (2), obesity (7) and/or cancer (2). Median duration of symptoms prior to CPI-006 was 8 days (range 1-21 days). No treatment-related adverse events were reported. There was no correlation between duration of symptoms and baseline anti-viral titers. Kinetics of anti-SARS-CoV-2 response to spike protein are shown for 7 pts with follow-up $\geq 7$ days post CPI-006 (figure 1). One pt with lymphopenia $(600 / \mathrm{mm} 3$ ) had delayed response to CPI-006; all other pts generated antibody response by Day 7 post-CPI-006 to both spike and RBD. Increasing titers of $\operatorname{IgG}$ and $\operatorname{IgM}$ antibodies were observed out to 28 days post treatment. In one pt examined, memory B cells increased from $1.81 \%$ to $4.83 \%$ of B cells 28 days after treatment with serum IgG titers to spike and to RBD of $>1: 50,000.2$ of 2 pts had increase in both CD4 and CD8 T effector memory cells at day 28. All pts were discharged (median 4 days) with clinical improvement.

Conclusions CPI-006 is well tolerated in COVID-19 pts. Low baseline titers of antibodies to virus were increased following CPI-006 in all treated pts. Immunomodulation with CPI-006 


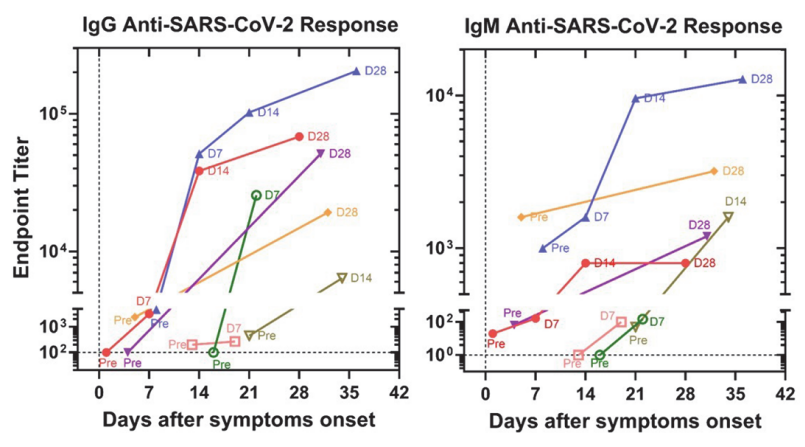

Abstract 325 Figure 1 Antibody responses to SARS-CoV-2 spike protein in patients treated with CPI-006

represents a novel therapy for COVID-19 aimed at stimulating more robust and prolonged anti-SARS-CoV-2 immunity potentially after infection or with vaccination.

Trial Registration NCT04464395

Ethics Approval The study was approved by Temple University Hospital's Ethics Board, Western IRB, approval number 11317457-1.

\section{REFERENCE}

1. Luke J, Powderly J, Merchan J, Barve M, Hotson A, Mobasher M, Kwei L, Luciano $G$, Buggy J, Piccione E, Miller R. Immunobiology, preliminary safety, and efficacy of CPI-006, an anti-CD73 antibody with immune modulating activity, in a phase 1 trial in advanced cancers. J Clin Oncol 2019; 37:15 suppl, 2505.

http://dx.doi.org/10.1136/jitc-2020-SITC2020.0325

\section{SARS-COV-2 SPECIFIC T-CELLS IN TIL FROM PATIENTS WITH EPITHELIAL CANCER}

${ }^{1}$ Pedro Noronha*, 'Georgia Paraschoudi, 'Eric Sousa, 'Jéssica Kamiki, 'Patrícia António, ${ }^{1}$ Carolina Condenço, ${ }^{1}$ Inês Silva, ${ }^{1}$ Andreia Maia, ${ }^{1}$ Mireia Castillo-Martin, ${ }^{1}$ António Beltran, ${ }^{2}$ Carlos Carvalho, ${ }^{1}$ Joana Lerias, ${ }^{3}$ Alimuddin Zumla, ${ }^{1}$ Markus Maeurer. ${ }^{1}$ Champalimaud Centre for the Unknown, Lisbon, Portugal; ${ }^{2}$ Champalimaud Clinical Centre, Lisbon, Portugal; ${ }^{3}$ University College London, London, UK

Background SARS-CoV-2 primarily infects the upper and lower airway system, yet also endothelial cells and multiple tissues/organ systems. Anti-SARS-CoV-2 directed cellular immune responses may be deleterious or may confer immune protection - more research is needed in order to link epitopespecific T-cell responses with clinically relevant endpoints. ${ }^{1}$ Analysis of epitope reactivity in blood from healthy individuals showed pre-existing $(\mathrm{CD} 4+)$ reactivity most likely due to previous exposure to the common old coronavirus species $\mathrm{HCoV}$ OC43, HCoV-229E, - NL63 or HKU1, or - not mutually exclusive - cross-reactive T-cell responses that would recognize SARS-CoV-2, yet also other non-SARS-CoV-2 targets. ${ }^{2,3}$ Detailed single cell analysis in PBMCs from patients with COVID-19 showed strong T-cell activation and expansion of TCR gamma - delta T-cells in patients with fast recovery or mild clinical symptoms. ${ }^{4}$ Previous studies examining antigenspecific T-cell responses in tumor-infiltrating T-cells (TIL) showed that EBV or CMV-specific cellular immune responses in TIL from patients with melanoma or pancreatic cancer. Such virus -specific T-cells may represent 'bystander' T-cell activation, yet they may also impact on the quality and quantity of anti-tumor directed immune responses. We tested therefore TIL expanded from 5 patients with gastrointestinal cancer, who underwent elective tumor surgery during the COVID-19 pandemic for recognition of a comprehensive panel of SARS-CoV-2 T-cell epitopes and compared the reactivity, defined by IFN-gamma production to TIL reactivity in TIL harvested from patients in 2018, prior to the pandemic. Methods A set of 187 individual T-cell epitopes were tested for TIL recognition using 100IU IL-2 and 100 IU IL-15. Different peptide epitopes were selected: i) all epitopes were not shared with the 4 common old coronavirus species, ii) some peptides were unique for SARS-CoV-2, and iii) others were shared with SARS-CoV-1. Antigen targets were either 15 mers or 9 mers for MHC class II or class I epitopes, respectively, derived from the nucleocapsid, membrane, spike protein, ORF8 or the ORF3a. The amount of IFN-gamma production was reported as $\mathrm{pg} / 10 \mathrm{e} 4$ cells/epitope/5 days. Controls included CMV and EBV peptides.

Results We detected strong IFN-gamma production directed against antigenic 'hotspots' including the ORF3a, epitopes from the SARS-CoV-2 nucleocapsid and spike protein with a range of 12 up to 30 targets being recognized/TIL.

Conclusions SARS-CoV-2 epitope recognition, defined by IFN production, can be readily detected in TIL from patients who underwent surgery during the pandemic, which is not the case for TIL harvested prior to the circulating SARS-CoV-2. This suggests a broader exposure of individuals to SARS-CoV-2 and shows that SARS-CoV-2 responses may shape the quality and quantity of anti-cancer directed cellular immune responses in patients with solid epithelial malignancies.

Acknowledgements We thank the Surgery, Pathology and Vivarium Units of Champalimaud Clinical Center (N. Figueiredo, A. Brandl, A. Beltran, M. Castillo, C. Silva ).

Ethics Approval This study was approved by the Champalimaud Foundation Ethics Committee.

Consent All donors provided written consent and the study was approved by the local ethics committee. The study is in compliance with the Declaration of Helsinki.

\section{REFERENCES}

1. Grifoni, A., Weiskopf, D., Ramirez, S. I., Mateus, J., Dan, J. M., Moderbacher, C. R., Rawlings, S. A., Sutherland, A., Premkumar, L., Jadi, R. S., Marrama, D., de Silva, A. M., Frazier, A., Carlin, A. F., Greenbaum, J. A., Peters, B., Krammer, F., Smith, D. M., Crotty, S., \& Sette, A. (2020). Targets of T Cell Responses to SARSCoV-2 Coronavirus in Humans with COVID-19 Disease and Unexposed Individuals. Cell, 181(7), 1489-1501.e15. https://doi.org/10.1016/j.cell.2020.05.015

2. Mateus, J., Grifoni, A., Tarke, A., Sidney, J., Ramirez, S. I., Dan, J. M., Burger, Z. C., Rawlings, S. A., Smith, D. M., Phillips, E., Mallal, S., Lammers, M., Rubiro, P., Quiambao, L., Sutherland, A., Yu, E. D., da Silva Antunes, R., Greenbaum, J., Frazier, A., ... Weiskopf, D. (2020). Selective and cross-reactive SARS-CoV-2 T cell epitopes in unexposed humans. Science, eabd3871. https://doi.org/10.1126/ science.abd3871

3. Le Bert, N., Tan, A. T., Kunasegaran, K., Tham, C. Y. L., Hafezi, M., Chia, A. Chng, M. H. Y., Lin, M., Tan, N., Linster, M., Chia, W. N., Chen, M. I.-C., Wang, L.-F., Ooi, E. E., Kalimuddin, S., Tambyah, P. A., Low, J. G.-H., Tan, Y.-J., \& Bertoletti, A. (2020). SARS-CoV-2-specific T cell immunity in cases of COVID-19 and SARS, and uninfected controls. Nature, 584(7821), 457-462. https://doi.org/ 10.1038/s41586-020-2550-z

4. Zhang, J., Wang, X., Xing, X. et al. Single-cell landscape of immunological responses in patients with COVID-19. Nat Immunol 2020;21:1107-1118. https:// doi.org/10.1038/s41590-020-0762-x

http://dx.doi.org/10.1136/jitc-2020-SITC2020.0326

\section{STUDY OF ANTI-PD-1 ANTIBODY MULTIMODAL COMBINATION AS FIRST-LINE TREATMENT ON TIME WINDOW OF ADVANCED SOLID TUMOR}

Jian Shi* ${ }^{*}$ Baoen Shan, Junyan Wang, Jiayin Liu, Rongfeng Liu, Yujie Shan. Fourth Hospital of Hebei Medical University, shijiazhuang, China 